\title{
Mathematical Optimization of the Tactical Allocation of Machining Resources for an Efficient Capacity Utilization in Aerospace Component Manufacturing
}

\author{
Sunney Fotedar ${ }^{1}$, Torgny Almgren ${ }^{2}$, Stefan Cedergren $^{3}$, Ann-Brith Strömberg ${ }^{1}$, and Michael Patriksson ${ }^{1}$ \\ ${ }^{1}$ Mathematical Sciences, Chalmers University of Technology, Gothenburg, Sweden \\ E-mail: sunney@chalmers.se, torgny.almgren@gknaerospace.com, stefan.cedergren@gknaerospace.com, \\ anstr@chalmers.se,mipat@chalmers.se, \\ ${ }^{2}$ Logistics, GKN Aerospace, Trollhättan, Sweden \\ ${ }^{3}$ PTC, GKN Aerospace, Trollhättan, Sweden
}

\begin{abstract}
In the aerospace industry, with low volumes and many products, there is a critical need to efficiently use available manufacturing resources. Currently, at GKN Aerospace, resource allocation decisions that in many cases will last for several years are to some extent made with a short-term focus so as to minimize machining time, which results in a too high load on the most capable machines, and too low load on the less capable ones. This creates an imbalance in capacity utilization that leads to unnecessary queuing at some machines, resulting in long lead times and in an increase in tied-up capital. Tactical resource allocation on the medium to long-range planning horizon (six months to several years) aims to address this issue by allocating resources to meet the predicted future demand as effectively as possible, in order to ensure long range profitability. Our intent is to use mathematical optimization to find the best possible allocations.
\end{abstract}

Keywords: Tactical resource allocation, Capacity utilization, Mixed Integer Linear Programming, Optimization, Manufacturing, Aerospace, Resource loading, Logistics, Multi-objective optimization.

\section{Introduction}

GKN Aerospace Engine Systems (GKN for short) in Trollhättan, Sweden, is a leading supplier of aircraft engine components. GKN's components have presence in almost all major commercial aircraft. The products produced in Trollhättan include casings and rotating parts for both the compressor and the turbine section of the engine. Rotation and a high temperature differences between different parts of the engine puts high, in many cases extreme, quality demands (tight tolerance limits) on the components. The capital-intensive production at a large aerospace tier-1 supplier like GKN is generally influenced by expensive materials, long supply lead times, a large product mix and demand variations (see [1]). The potential reduction of tied-up capital that can be achieved by decreasing lead times is therefore substantial; in addition the project is expected to increase the overall resource utilization.

\subsection{Production context}

Manufacturing is performed in multiple steps, such as cutting (milling, turning, drilling, and grinding), welding, assembly, heat/surface treatments, and control/measurements. For cutting, GKN has a variety of production resources (machines) with different functions. The factory is organized in several functionally oriented production shops, and most of the pro- duction resources are shared by different products. Each production shop is organized as a job-shop, where similar types of machines are placed in proximity to each other. A complication is that it is, in practice, impossible to physically move machines, as they are bulky and fixed into the ground in 2-5 meter deep pits. Thus, the factory as such can only to a very limited extent be adapted to changes in the product mix; it is therefore not possible (highly unlikely) to maintain perfect flows of parts through the factory over time.

\subsection{New product introduction}

The process of introducing a new product is tied-up with tactical resource allocation decisions. These tactical resource allocations (which are referred to in this text) should not be mistaken for the short-term resource allocations done when choosing between several available machining resources while scheduling. The latter is commonly addressed in industry (see an example from GKN, [2]). The tactical resource allocations identify which production processes that need to be developed. The processes developed then constitute the solution space for the short-term allocations, by deciding which resources are available for allocation for the short-term planning. The tactical problem therefore differs significantly from the short-term planning problem. 
The development of the required production processes consists of a number of activities that are needed to get the required approvals from customers and authorities in order to make a resource available for a specific operation on a product. The technical preparations (also called qualifications) often require several days of computer numeric control (CNC) programming, design and production of new fixtures, testing and validation, etc. These activities are the main parts of an iterative process to acquire the customer approval, and may take several months to complete. The time and costs associated with this process is often significant; the initial resource allocations are therefore seldom changed. However, if forced changes are made due to large variations in product demand and/or changes in available capacity of machines, it leads to delays and delivery disturbances. The tactical resource allocations will thus have a long-lasting effect on delivery times, product profitability and efficiency of the production system. Thus, it is of utmost importance that the resource allocations are made in an optimal way, so that changes in the later stages can be avoided.

The first step in the process to introduce new products is the mapping of the new product to a suitable value stream. Different products, or part types (sometimes a product is made of multiple part types), belonging to the same value stream are quite similar in terms of functionality. For example, all rotating parts should be in the same value stream. The reason is that it is easier to control geometrical tolerances by analyzing the data on machines producing similar products. This data is stored in the statistical process control (SPC) systems at GKN. Studying the previous SPC results has been one way of analyzing the suitability of a certain machine to handle new parts, by studying historical process outcome on similar features, such as a hole. Then experts apply the tolerance limits from the new part to get an understanding of how the machine would perform when introducing this part. This is easier if the newly introduced part is similar to the once already being machined. So, to get an overview on all the machining-related characteristics for different machines is possible. These issues are of the highest importance in the aerospace industry, as any regular occurrence of non-conformance (i.e., a slight deviation from a customer tolerance limit) results in halting of production.

Once a value stream is identified for the product, an operations list is created by the manufacturing experts. The operations list contains the operations needed to produce a certain product (or part), as well as the specific machine in which the respective operation must be performed. The capacity planners who are responsible for managing and determining production capacity are involved to check if the capacity is available in these machines. At this stage, however, no detailed assessment of the impact on resource allocation of varying demand for products is made. This is the reason that sometimes, when there are changes in the demand of some products or capacity of some machines, it may result in an additional effort to change some product routings which requires waiting for customer approval. Thus, the resource allocation plan should be robust such that the routing is still feasible, even when demand or capacity has varied slightly from their respective ex- pected values.

\subsection{The need for tactical resource allocation}

Internal company analyses have identified queuing at machines as a main contributor to long production lead times (see [1, p. 66]). As the queues are often caused by uneven loads on the production resources (see [3]), the production system must be prepared for such unavoidable demand variations, i.e., the resources need to have some buffer capacity. Due to accounting principles, the preliminary resource allocations often have a misleading impact on the internal cost assessment (see [4]), which often results in sub-optimal decisions from an overall capacity planning perspective (i.e., fast resources are favored, leading to an unbalanced resource loading). Therefore, a long-range tactical resource allocation is essential to achieve the required flexibility to absorb these variations, and to avoid unnecessary queuing.

This leads us to the research questions to be discussed in the following sections. We need to identify an objective function which will result in product routings with desirable properties, and in a later phase, good resource allocations. These properties constitute a balanced distribution of work load between machines; the robustness of the final allocation (minimum reallocations between time-periods); high machining standards; and a minimum distance travelled by products/part types between different physical areas in the factory. The relative importance of these objectives may vary, and it is an area of policy discussions for GKN. However, a balanced capacity utilization and high machining standards are the two most important properties.

\subsection{Research front}

There are rather few studies focused on the tactical resource planning problem on the medium- to long-range planning horizon. Tactical planning in general is a tool that can be used for reducing the effect of uncertainties in the production environment, and assist in creating stability in the production system (see [5,6]). One of the reasons why this type of optimization model has been developed with limited success in few cases is due to the computational complexity of the problem. From a combinatorial perspective, there are so many feasible solutions to evaluate that the computation times tend to be so long that the problem has historically been considered practically insoluble. Our research aims to utilize optimization theory to solve such models in a reasonable time frame. There are not many industrial scale tactical resource allocation problem solved from a mathematical optimization perspective. This type of approach provides, not only a feasible solution, but also a measure of goodness (the optimality gap, i.e., the ratio of the difference between the best known solution and a value that bounds the best possible solution to the best known solution) (see the full definition in [7]). Most of the commercial software used to our knowledge either use heuristics or some other inexact methods lacking this feature. However, there are commercial mathematical optimization solvers such as Gurobi [8] and CPLEX [9], which can be used to solve such problems, but it requires identifying the 
right objective function and constraints, and also modelling them in an efficient way. This is also a part of what we do.

\section{Scope and problem description}

The scope of the proposed tactical resource allocation model is restricted to allocating products to machines which perform cutting operations. They includes milling, drilling, grinding, and turning machines. The model should ideally be used when a new product is introduced, or when there are significant changes in the machining capacity (e.g., decommissioning of machines or the introduction of new machines). The proposed model should be used by the capacity planner and by product introduction leaders at the company.

Each product has a bill of material containing details about the product structure, including all the part types, and their quantities used in the final product. However, it is quite common that products are composed of one part. Each product/part type has an operations list, i.e., a list of manufacturing operations, such as milling, drilling, turning, and grinding, required to manufacture this product/part. A combination of a product/part and such a specific operation constitutes a job type. The task of the resource allocation model is to assign each job type to resources (machines) such that the imbalance in capacity utilization is minimized. A simple example with five machines and a part type having three operations is illustrated in Fig. 1. We have a single part (Part 1) which requires three operations to be completed, and let us assume all five machines are capable of doing these three operations. We also define a user parameter $\tau$ which is the maximum number of machines which are allowed to do a specific job type, i.e., only machines 1 and 5 are allowed to first operation on the first part in Fig. 1. It is desired to identify only few machine alternatives for each job type or a user defined parameter $(1 \leq \tau \leq 5)$. This restriction is to prevent too complicated flows and limit the number of qualifications or tests to be conducted by manufacturing experts to check the performance of a machine for a particular type of operation on a specific part. One possible solution (for $\tau=2$ ) are qualifying machines 1 and 5 for the first operations, 3 and 4 for the second and 2 and 5 for the third operation. This results in the following eight possible routings $1-4-2,1-4-5$, $1-3-2,1-3-5,5-3-2,5-3-5,5-4-5$, and 5-4-2. So, if we look at this small combinatorial problem, we started with $\left(\left(\begin{array}{l}5 \\ 2\end{array}\right)\right)^{3}=10^{3}$ alternative routings, and we selected only $2^{3}$ which results in capacity balancing. Moreover, typically there are approximately 3-4 part types in a new product and each have at least 3-5 operations, and there are nearly 150 machines. Thus, a simple enumeration for such a large instance of the problem is too computationally time-consuming and we need to use mathematical optimization to find the best possible allocations. Thus, in a way optimization helps manufacturing experts to identify which machines to qualify, such that both qualification costs and capacity imbalance are minimized for the next 3-5 years.

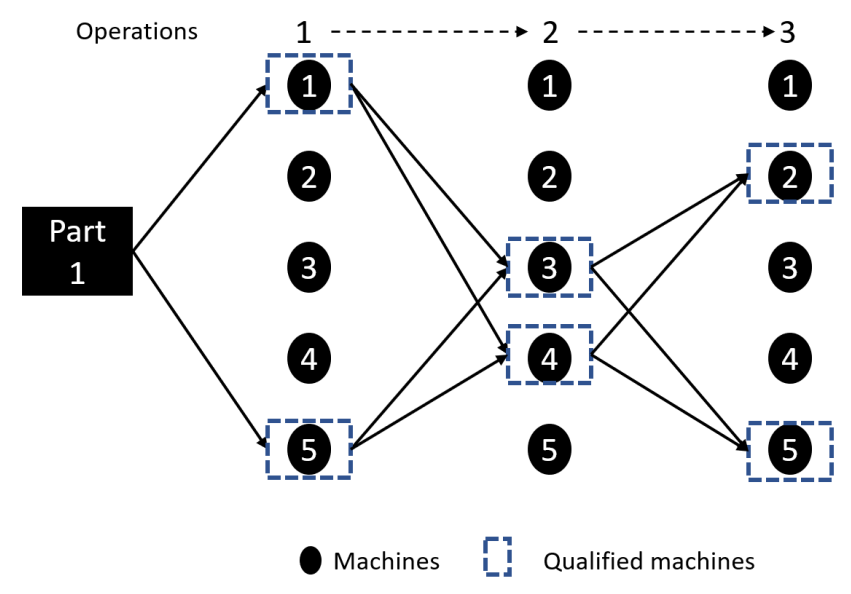

Figure 1: Part routes

\subsection{Constraints}

All product routings must satisfy some constraints. According to an internal company report, the constraints are classified into hard and soft constraints. Hard constraints are also referred to as level 1 constraints, while soft constraints are divided into level 2 and level 3 constraints. Going from level 1 to level 3, the importance of the constraints decreases (see Fig. 2). In Fig. 2, the base represents the size of the set of possible allocations of machines to orders of different job types; this set reduces as we add restrictions on, e.g., the number of qualifications (or qualification cost). The vertical position in Fig. 2 represents relative importance. In optimization parlance, the violation of soft constraints could be either combined and added as a penalty in the objective function (see [10]), which is to be minimized, or treated/modeled as different criteria in a multi-criteria optimization problem (see [11]).

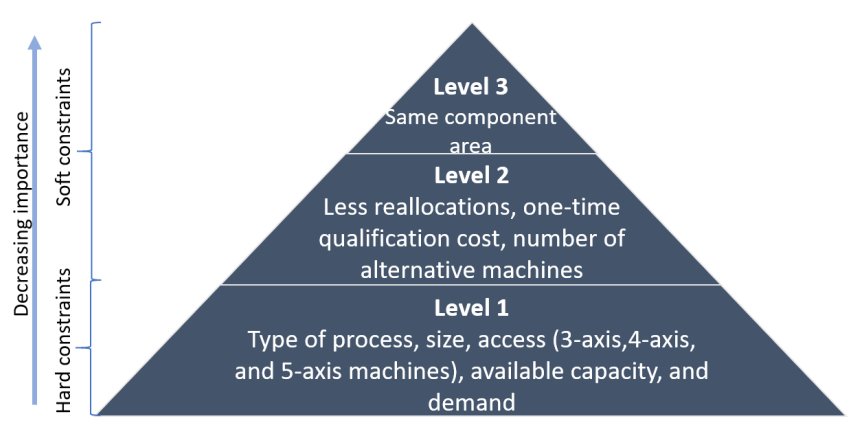

Figure 2: Constraints

Hard constraints Hard constraints are those which must be satisfied during each time-period within the long-range planning horizon. These constraints include a required compatibility between job type and machine, based on the type of process (milling, turning, etc.) and whether a job type of a given dimension (e.g., diameter) can be processed on a given machine (i.e., size compatibility). Lastly, the projected workload on a machine should be less than or equal to its total 
capacity, and the demand for each job type should be met in each time-period.

Soft constraints It is not mandatory to satisfy constraints which are associated with the preferences of the capacity planners. The constraints belonging to level 2 are mentioned below.

1. Reallocations: Preparing a machine for a job type is a resource-demanding activity; therefore, it is prudent to avoid changing the routing of orders of a given job type too often. For example, if we represent a job type as $(i, j)$, where $i \in$ PartTypes, and $j \in$ Operations, then a binary decision variable $s_{i j k}^{t}$ can be used to represent whether an order of job type $(i, j)$ is processed in machine $k \in$ Machines at time-period $t \in$ TimePeriods. The value of the variable $s_{i j k}^{t}$ is 1 , if job type $(i, j)$ is performed in machine $k$, otherwise, it is equal to zero. Now, we use the introduced variables to clarify when a reallocation is considered. In Table 1, we highlight three alternative allocations (three rows) for machine 2 for the resource allocation sub-problem with one job type $(1,1)$, and for five time-periods. Let us assume that all three allocations are feasible (that is, they satisfy the level 1 constraints). A reallocation of job type $(i, j)$ to machine $k$ at time $t$ is determined by the variable values $s_{i j k}^{t-1}=0$ and $s_{i j k}^{t}=1$ (i.e., at the consecutive time periods $t-1$ and $t$ ). The first solution in Table 1 comprises one reallocation at $t=5$, while the second comprises two reallocations at $t=3$ and $t=5$, respectively. The third solution comprises no reallocations and it is therefore preferred. This reasoning is valid provided there are no reallocations at other machines.

Table 1: Reallocations between time-periods

\begin{tabular}{cccccc}
\hline$s_{112}^{1}$ & $s_{112}^{2}$ & $s_{112}^{3}$ & $s_{112}^{4}$ & $s_{112}^{5}$ & Reallocations \\
\hline 0 & 0 & 0 & 0 & 1 & 1 \\
\hline 0 & 0 & 1 & 0 & 1 & 2 \\
\hline 1 & 1 & 1 & 0 & 0 & 0 \\
\hline \hline
\end{tabular}

2. One-time qualification: Generally, for any new product, there is a first-time qualification of each machine to which it is assigned. However, for orders of job types related to existing products, there exist machines which are already qualified or used historically. Nevertheless, whenever reallocating orders of an existing product to another machine, which is technically capable but not qualified before, a one-time cost for qualifying the other machine for that product arises. This means additional man-hours for programming $\mathrm{CNC}$ or developing new fixtures, and sometimes waiting for the customer approval. It is preferred to keep this fixed cost as low as possible. However, it is to be checked whether allowing qualifications results in an improved capacity balancing.

The level 3 constraints are the least important. One of the con- straints included is the limitation of the total distance travelled by orders. Each machine is assigned to a physical space in the factory, so-called component area in the factory. It is desired to keep the number of times orders must flow in-between these component areas below a pre-defined number, or to keep it as low as possible. This helps to maintain an efficient flow and to reduce internal transportation.

\subsection{Optimization model}

The approach taken to solve this resource allocation problem is to utilize a mathematical optimization model. The output of the proposed optimization model is the assignment of the different job types to the machines. The model identifies a small number of different product routings for the capacity planners/short-term scheduling. We plan to obtain a resource allocation for a period of three to five years, with quarterly or monthly time-periods. The demand for products is steady, and there is a steady flow of raw materials through the factory. An optimization model requires an objective function which assesses the quality of the solutions, while a set of constraints to restrict the possible allocations, which in turn restricts the achievable objective value. We have mentioned all the constraints in the previous section. Thus, next we discuss how to incorporate the desired properties of the final resource allocation plan into the objective function.

1. Objective function: There are some articles which have discussed approaches to calculate internal production costs for products in similar production environments (see [4, 12, 13]). Most of these articles have considered product cost as a function of the total machining hours spent on a product. This is the legacy of the traditional accounting approach as mentioned in [4]. They use the product routing as a constant in their cost function, but with our approach we let the product routing be a variable, and instead of minimizing machining hours we minimize resource utilization imbalance. This is to our knowledge a novel approach and much more effective in improving flow efficiency in functional oriented production. We define utilization as the ratio of planned hours of machining to total available hours in a machine. Thus, to obtain a balanced utilization, we propose minimizing the (planned) utilization which is above a threshold value, that is, imposing a penalty for each time period when a machine's planned utilization is above a predefined threshold $\zeta$, where $0 \leq \zeta \leq 1$.

2. Decision variables: To define the objective function, we introduce some notation. We represent the number of orders of type $(i, j)$ produced in time-period $t$ in machine $k$ by $x_{i j k}^{t}$. The capacity (in hours) of machine $k$ is denoted by using capacity $C_{k} \geq 0$ (Note: In case of planned decommissioning of machines capacities can be indexed over time). The number of hours required to process an order of job type $(i, j)$ in machine $k$ is represented by $p_{i j k}$ (also including average set-up time as we do not assume batch production). In (1), the inner bracket represents the total resource loading of machine $k$ during time-period $t$. Thus, dividing it by the total capacity of 
machine $k$ yields the relative utilization at time-period $t$. The plus-operator, denoted $[\cdot]_{+}$sets any negative value to zero. Our first proposed objective function to be minimized over the set $X$ of feasible solutions satisfying the level 1 constraints is defined as to

$\operatorname{minimize} z_{1}(x \in X):=\sum_{t} \sum_{k}\left[\frac{1}{C_{k}}\left(\sum_{i} \sum_{j} p_{i j k} x_{i j k}^{t}\right)-\zeta\right]_{+}$

Our second alternative for an objective function is defined as to

$\operatorname{minimize} z_{2}(x \in X):=\sum_{t} \max _{k}\left[\frac{1}{C_{k}}\left(\sum_{i} \sum_{j} p_{i j k} x_{i j k}^{t}\right)-\zeta\right]_{+}$.

The function $z_{1}$ represents the average of non-negative excess capacity utilization (summed over the machines and the time-periods). The function $z_{2}$ represents the maximum (over the machines) excess utilization (summed over the time-periods). Since the average can be composed by both small and (very) large values, the function $z_{2}$ represents over-utilization more adequately than $z_{1}$. A review of such inequity-averse optimization approaches is described in detail in [14]. The benefits of using the (2) are also highlighted in the work done on resource allocation problems in [15].

\section{Expected results and future research work}

The proposed optimization model is a mixed-integer linear programming model. The objective function and constraints are linear or made linear using integer/binary variables. The goal of the optimization model is to produce optimal or nearoptimal solutions in a reasonable time frame. Since we are not aware of the lower limit (best possible feasible values) of some of the soft constraints, we intend to use one of the iterative frameworks as mentioned in [11].

\subsection{Expected results}

The proposed decision model should enable a faster and better adaptation of the production system to changes in the market and production conditions. Using our model, the organization's aim is to increase the available machining capacity by at least $2 \%$, which could mean a 4 -digit number of additional hours of more machining time available each year. These additional machining time could be made available when new products are introduced, or when the demand for some of the existing products increases. It is expected to reduce the number of over-utilized machines, which will result in reduced queues and, subsequently, reduced lead times.

\subsection{Future research work}

We intend to use real production data extracted from the Enterprise resource planning (ERP) system at GKN. We will identify an existing product and consider it as a new product to be introduced in the factory to test the model. We will utilize the historical resource allocation to find out which machines are qualified to perform operations on the existing products. We also plan to take input from manufacturing experts from GKN on identifying other similar machine alternatives which can perform some of these operations. This will increase the size of the feasible set of possible allocations, which is important for improving the optimal value. We will perform computational experiments to benchmark different model formulations with respect to computation time and solution quality.

\section{References}

[1] A.J. Lewestam and H.S.U. Mäki. Increasing throughput potential in functional oriented machining plants. Master Thesis, Division of Logistics and Transportation, Chalmers University of Technology, Göteborg, Sweden, 2015.

[2] K. Thörnblad, A.-B. Strömberg, M. Patriksson, and T. Almgren. Scheduling optimisation of a real flexible job shop including fixture availability and preventive maintenance. European Journal of Industrial Engineering, 9(1):126-145, 2015.

[3] E.M. Goldratt. Computerized shop floor scheduling. International Journal of Production Research, 26(3):443$455,1988$.

[4] A. Myrelid and J. Olhager. Hybrid manufacturing accounting in mixed process environments: A methodology and a case study. International Journal of Production Economics, 210:137-144, 2019.

[5] J. Olhager and E. Selldin. Manufacturing planning and control approaches: market alignment and performance. International Journal of Production Research, 45(6):1469-1484, 2007.

[6] A.M.T. Thomé, L.F. Scavarda, N.S. Fernandez, and A.J. Scavarda. Sales and operations planning: A research synthesis. International Journal of Production Economics, 138(1):1-13, jul 2012.

[7] Optimality gap: https://glossary.informs.org/ ver2/mpgwiki/index.php/Optimality_gap.

[8] Gurobi's website: https://www.gurobi.com/

[9] IBM's CPLEX optimizer: https://www.ibm.com/ analytics/cplex-optimizer.

[10] M.L. Fisher. The Lagrangian relaxation method for solving integer programming problems. Management Science, 50(12):1861-1871, 2004.

[11] R.T. Marler and J.S. Arora. Survey of multi-objective optimization methods for engineering. Structural and Multidisciplinary Optimization, 26(6):369-395, 2004. 
[12] C. Windmark and C. Andersson. Cost assessment of a production system - a method targeting a product's aggregated value stream costs. Procedia Manufacturing, 25:231-238, 2018.

[13] E.W. Hans, W. Herroelen, R. Leus, and G. Wullink. A hierarchical approach to multi-project planning under uncertainty. Omega, 35(5):563-577, 2007.

[14] Ö. Karsu and A. Morton. Inequity averse optimization in operational research. European Journal of Operational Research, 245:343-359, 2015.

[15] H. Luss and D.R. Smith. Resource allocation among competing activities: a lexicographic minimax approach. Operations Research Letters, 5(5):227-231, 1986. 